\title{
Identificação de rotavirus associado ao sorotipo G2 em Yucatan, México
}

\author{
Identificacion of rotavirus associated to seroty \\ G2 in Yucatan, Mexico
}

María del R. Gonzales-loza, Giraldo G. Polanco-Marín e Marylin Puerto-Solis

\begin{abstract}
Resumo Neste estudo reporta-se a identificação de rotavirus sorotipo G2 proveniente de amostras fecais de crianças com gastroenterite, da Cidade de Mérida, Yucatán, México. O diagnóstico virológico foi feito através da eletroforese em gel de poliacrilamidia e ensaio imunoenzimático. Das 149 amostras estudadas, 25 (16,7\%) foram positivas para rotavirus do grupo A; dessas 23 (92\%) foram sorotipo G2, subgrupo I e padrão eletroforético curto e 2 (8\%) subgrupo II e padrão eletroforético longo, porém, não foi possível determinar o sorotipo G. Desde 1985, até hoje, foi a única vez que o sorotipo G2 foi identificado, em mais de $90 \%$ das amostras analisadas. Acreditase que, provavelmente, nos últimos anos tem acumulado na população, um grupo de pessoas suscetíveis ao sorotipo de RV, e num futuro poderia ter um surto importante de gastroenterite, associado ao sorotipo G2 de rotavirus.
\end{abstract}

Palavras-chaves: Rotavirus. Sorotipo G2. Gastroenterite.

Abstract In the present study, rotavirus G2 serotype was identified from fecal samples of children with gastroenteritis from the city of Merida, Yucatan, Mexico. Virological diagnosis of disease was performed using polycrylamide gel electrophoresis and immunoenzymatic assay. Out of 149 analyzed samples $25(16.7 \%)$ gave positive reaction to rotavirus groups $A$, of these $23(92 \%)$ were identified as serotype g2, subgroup $i$ and electrophoretic short pattern, whereas 2 (8\%) were identified as subgroups II and electrophoretic long pattern, however, the G serotype was not possible to determine. Rotavirus $G$ serotype has not been detected in more than $90 \%$ of samples since 1985. This indicates that the number of people susceptible to G2 serotype within the population has increased over recent years, which perhaps indicates that an important outbreak of acute infectious diarrhea caused by the rotavirus G2 serotype may be forthcoming.

Key-words: Rotavirus. G serotype. Gastroenteritis.

O grupo $A$ de rotavirus $(\mathrm{RV})$ é a causa mais comum de gastroenterite aguda em crianças de todo o mundo. Nos países em desenvolvimento estima-se que, anualmente, ocorra mais de 870.000 mortes, em crianças menores de 5 anos $^{1024}$.

Os rotavirus contêm um genoma de 11 segmentos de RNA de dupla cadeia, envolvidos em três camadas de proteínas ${ }^{5}{ }^{12}$. Os pesos moleculares dos 11 segmentos têm servido para sua classificação, de acordo com seu padrão de migração no gel de poliacrilamida. Deste modo, temos que os RV se classificam em padrões curtos e longos, dependendo da migração do segmento $10^{116}$.

A camada externa destes vírus está formada por duas proteínas (VP4 e VP7) que possuem epítopos que induzem à formação de anticorpos neutralizantes ${ }^{26}$.

\footnotetext{
Laboratório de Virología, Centro de Investigaciones Regionales "Dr. Hideyo Nouguchi”. Merida, Yucatán, México.

Address to: Dra. María Gonzáles Losa. Laboratório de Virología, Centro de Investigaciones Regionales "Dr. Hideyo Nouguchi". Av. Itzáez por 59, 490, Centro, 97000 Merida, Yucatán, México.

Tel: 99246412 ext 137; fax: 99236120

e-mail: glosa@tunku.uady.mx

Recebido para publicação em 15/7/99.
} 
Na camada interna encontra-se a proteína VP 6 , à qual possui determinantes antigênicos de subgrupo, que classificam os RV em I, II, I/II, e não I/II, sendo o subgrupo II o mais freqüente a nível mundial'.

Em 1989, Estes e Cohen propõem um sistema de classificação para os rotavirus derivados das duas proteínas do capsidio viral: os sorotipos $\mathrm{G}$, com base na glicoproteína VP7, que é a mais abundante, e os sorotipos $P$, que são definidos por epítopos na proteína VP4, sensível à protease $^{212}$. Atualmente, utilizando testes de neutralização, foram descritos 14 sorotipos $G$ e 20 sorotipos $P$ em humanos e animais. Porém, apesar da diversidade de sorotipos $G$ encontrados em humanos (G1, 2, 3, 4, 5, 6, 8, 9, 10 e 12), a maioria das infecções sintomáticas está associada aos sorotipos G 1-4 e destes o 1, é o que se encontra com mais freqüência no mundo ${ }^{1142023}$.

Trabalhos realizados em diferentes partes do mundo têm demonstrado que os sorotipos $\mathrm{G}$ mais freqüentes são o 1,3 e 4, os quais distribuem-se de modo endêmico. Ademais, sabe-se que o G2 se apresenta em menor freqüência e de forma epidêmica ${ }^{149}$.

Em 1998, Padilla et al reportam um estudo realizado em três diferentes regiões do México para conhecer a distribuição e freqüência dos sorotipos $\mathrm{G}$ de rotavírus que circularam durante dois anos consecutivos no país. Quarenta e cinco porcento das amostras foram de sorotipo G3, $22 \%$ G1 , G2 somente foi detectado, no centro e sudeste do país, em 7\% das amostras e 0 sorotipo G4 esteve presente em todo o país em $1 \%$ de todas as amostras ${ }^{16}$

No Estado de Yucatán, o rotavírus é uma importante causa de morbidade: já que se encontra associado a mais de $50 \%$ dos casos de diarréia infecciosa aguda (DIA) durante os meses de inverno ${ }^{3}$. O estudo dos sorotipos $\mathrm{G}$ de rotavírus circulantes em Yucatán tem demonstrado a presença dos 4 sorotipos $\mathrm{G}$ mais importantes, observando-se que as epidemias são predominantemente de um só sorotipo, que varia segundo 0 ano ${ }^{818}$.

O conhecimento da epidemiologia dos sorotipos $G$ de $R V$, em diferentes partes do mundo e em diferentes tempos, é necessário para poder valorizar qual é a distribuição dos mesmos e as mudanças que vão sofrendo com o tempo, para se definir as caraterísticas antigênicas das cepas que predominam em cada população. Este conhecimento constitui a base da vigilância epidemiológica da DIA por RV.

Neste trabalho, descrevemos a identificação de cepas de rotavírus de padrão curto e serotipo G2 em crianças com menos de cinco anos de idade, com gastroenterite aguda, internadas por desidratação severa, na cidade de Mérida, Yucatán, México.

\section{MATERIAL E MÉTODOS}

Trata-se de um estudo transversal, no qual se pesquisaram todas as crianças com idade inferior a 5 anos, internadas por diarréia infecciosa, com menos de 5 dias de evolução, no Pronto Socorro do Hospital General O'Horán, de Mérida, Yucatán, durante o período de $1^{\circ}$ setembro de 1989 a 28 de fevereiro de 1990.

Coletaram-se amostras de fezes nas primeiras 24 horas de hospitalização de todas as crianças que preenchessem os critérios de inclusão. As amostras foram levadas ao laboratório e processadas para o diagnóstico de RV, no mesmo dia da coleta; as amostras positivas foram armazenadas a $-20^{\circ} \mathrm{C}$ para determinação de sorotipos, subgrupos e padrão eletroforético.

Para o diagnóstico de RV utilizou-se em todos os casos, a técnica da eletroforese do RNA viral, em gel de poliacrilamida (PAGE) com solução de nitrato de prata e ensaio imunoenzimático (ELISA), para detectar grupo A de RV ${ }^{8}$.
Para designar o subgrupo e o sorotipo realizou-se a técnica de ELISA com anticorpos monoclonais contra os subgrupos I e II e contra os quatro sorotipos $\mathrm{G}$ mais importantes, doados pelo Dr. Harry Greenberg (Veterans Hospital de Palo Alto, CA). Os monoclonais utilizados foram os seguintes: $255 / 60$ subgrupo I, $631 / 9$ subgrupo II, o KU4 e o SE8 contra o sorotipo 1; o S2G10, 2F1 e IC10 contra o sorotipo 2; o YOCE2, 159 y 4F8 contra o sorotipo 3 e STG11 contra o sorotipo 4.

A metodologia utilizada foi, resumidamente, a seguinte: as imunoplacas (NUNC, Dinamarca) foram sensibilizadas com os diferentes monoclonais diluídos 1/1000 em PBS e incubadas por 24h a $4^{\circ} \mathrm{C}$; posteriormente, foram lavadas e bloqueadas com albumina sérica bovina (BSA) a 1\% em PBS, por mais $24 \mathrm{~h}$ a $4^{\circ} \mathrm{C}$. Ao final desse tempo, as placas foram lavadas novamente e se agregaram às amostras fecais diluídas 1/10 em PBS-BSA a 5\% e incubadas a $37^{\circ} \mathrm{C}$ por duas horas. Posteriormente, foram incubadas com um anticorpo policlonal de 
coelho contra rotavirus diluído em PBS/BSA a $0,5 \%$, e incubadas novamente a $37^{\circ} \mathrm{C}$ por duas horas. Logo após, foram lavadas e incubadas com anti IgG de coelho marcado com peroxidase (Sigma, USA) por uma hora a $37^{\circ} \mathrm{C}$, para logo adicionar o substrato OPD (Sigma, USA), a reação foi interrompida com ácido sulfúrico $2 \mathrm{M}$ e interpretada num leitor de ELISA (Behring) com um filtro de $492 \mathrm{~nm}$ de comprimido de onda. As amostras cuja absorvância foi duas vezes maior que a dos controles negativos foram consideradas positivas.

As amostras que reagiram com dois ou mais sorotipos, somente foram consideradas como de um sorotipo, quando a absorvância de um deles foi duas vezes maior que a de qualquer dos outros sorotipos. Como controle positivo, utilizaram-se as seguintes cepas: WA, DS1, ST e ST3.

\section{RESULTADOS}

Durante os seis meses do estudo coletaramse 149 amostras de fezes de crianças com menos de cinco anos de idade internados com DIA, todas elas com desequilíbrio hidroeletrolítico moderado ou severo, o qual foi o motivo da internação.

Das 149 amostras estudadas $25(16,7 \%)$ foram positivas para RV, sendo que $23(92 \%)$ foram positivas em ambos métodos e 2 (8\%) só por eletroforese.

Das 25 amostras positivas 23 (92\%) apresentaram padrão eletroforétito curto e $2(8 \%)$ padrão longo, nas 2 positivas somente pela eletroforese, uma era de padrão longo e um de padrão curto. Quanto à caracterização do subgrupo, as 23 amostras de padrão curto reagiram com o monoclonal de subgrupo I e as duas de padrão longo com o do subgrupo II. Os resultados da ELISA para caracterizar o sorotipo $G$ foi o seguinte: das 23 amostras com o padrão curto e subgrupo I, 18 (81,8\%) foram sorotipo G2, das 5 amostras restantes, $2(9,1 \%)$ reagiram com todos os monoclonais utilizados, $2(9,1 \%)$ não reagiram com nenhum e em 1 não se realizou este teste. As amostras de padrão longo não puderam ser sorotipadas já que não reagiram com nenhum dos monoclonais utilizados.

\section{DISCUSSÃO}

A distribuição dos sorotipos $\mathrm{G}$ de rotavirus numa determinada população, é parte importante da vigilância epidemiológica. A partir de 1985, após iniciar o trabalho de sorotipagem de RV em nosso Estado, é que se pode constatar a presença dos quatro sorotipos $\mathrm{G}$ mais comuns, mas, sempre com predominância de só um. Durante os primeiros cinco anos de estudo, o sorotipo 4 foi o que se apresentou com mais freqüência. Posteriormente, aparece o sorotipo G3 em mais de $50 \%$ do total das amostras e nos últimos dois anos o sorotipo G1 é o que tem predominado nas epidemias $^{816}$. O sorotipo G2 foi identificado pela primeira vez no período entre 1989 e 1990 e desde então, tem-se encontrado todos os anos com uma freqüência sempre menor que $10 \%$ e sem nenhum padrão epidemiológico caraterístico.

A nível nacional, tem se descrito predominância dos sorotipos $\mathrm{G} 1$ e 3 e uma baixa freqüência do G2 $(7 \%)$ no Centro e Sudeste do pais ${ }^{16}$.

A distribuição dos diferentes sorotipos $G$ no País e no Estado é similar ao reportado a nível mundial ${ }^{1}$.

Das cepas aqui descritas, $81,8 \%$ guardam as características antigênicas clássicas dos RV, já que estas 18 amostras tiveram padrão eletroforético curto, subgrupo I e sorotipo G2. Estas correlações eletroforéticas e antigênicas têm-se perdido com o aparecimento de novas cepas de padrão curto que não reagem de maneira específica com os monoclonais de sorotipo G2 ${ }^{1}$.

Em nosso estudo, 4 (18,2\%) amostras não reagiram com nenhum dos monoclonais utilizados, 2 tiveram padrão curto e subgrupo I e 2 padrão longo e subgrupo II.

Recentemente, tem-se descrito a presença de cepas de rotavirus com serotipos $G$ pouco usuais, em fezes de crianças com diarréia, em diferentes países e que, ao que parece, provêm de rearranjos entre humanos e animais. Na Índia, a partir de 1995, apareceram cepas que não puderam ser classificadas com os anticorpos monoclonais para sorotipos G 1-4 e que pertencem ao sorotipo G9. Nos Estados Unidos reportou-se que o sorotipo G9 tem sido importante nas últimas epidemias. No Egito e na Austrália identificaram-se cepas com sorotipo G8 e no Brasil com sorotipo G5 101113151721 .

Os resultados mencionados anteriormente nos obrigam a pensar na possibilidade de que as 4 amostras que não reagiram com nenhum monoclonal poderiam ter um sorotipo $G$ pouco 
usual, o qual não pôde ser determinado com a variedade de monoclonais utilizada. Por isto fazse necessário implementar outros métodos de classificação dos RV, como é a genotipagem para utilizá-la de forma rotineira na vigilância epidemiológica.

As cepas de RV que pertencem aos sorotipos G1, 3 e 4 apresentam caraterísticas antagônicas na VP6 e VP4 já que pertencem ao subgrupo II e sorotipo P1. Isto faz com que a infecção com um deles induza proteção cruzada parcial aos outros sorotipos resultando que infecções posteriores apresentem um quadro clínico leve ou infecções assintomáticas. O sorotipo G2 não compartilha determinantes antigênicos em VP6 com os outros sorotipos, pois pertence ao subgrupo I e quanto à VP4, geralmente, encontrase associado a P2. Assim nas três proteínas mais importantes na indução de anticorpos, as cepas G2 são diferentes das $\mathrm{G} 1,3$ e 4, pelo que, aparentemente, a infecção com algum dos outros sorotipos não induz proteção contra este sorotipo ${ }^{15} 2022$.

Pelas caraterísticas antigênicas e epidemiológicas das cepas de rotavirus do sorotipo G2, é provável que ao longo destes últimos anos tenha-se acumulado um grupo de pessoas suscetíveis ao sorotipo G2 e que, em algum momento surja um surto de DIA por este agente, o que nos obriga a continuar e estender a vigilância epidemiológica dos $\mathrm{RV}$ em nossa cidade.

\section{REFERÊNCIAS BIBLIOGRÁFICAS}

1. Aijaz S, Gowda K, Jagannath HV, Reddy RR, Maiya PP, Ward RL, Greenberg HB, Raju M, Babu A, Rao C. Epidemiology of symptomatic human rotaviruses in Banglore and Mysore, India, from 1988-1994 as determined by electrophorotype, subgroup and serotype analysis. Archives of Virology 141:715-726, 1996.

2. Alferi A, Leite J, Nakagomi O, Kaga E, Woods P, Glass R, Gentsh J. Characterization of human rotavirus genotype $\mathrm{P}$ (8) G5 from Brazil by probe hybridization and sequence. Archives of Virology 141:2353-2364, 1996.

3. Baeza-Bacab M, Polanco-Marín GG, Puerto-Solís M, González-Losa MR, Manzano-Cabrero L, Pino-Rosado JM. Cambios en el comportamiento endémico de la diarrea causada por rotavirus en el estado de Yucatán, México. Informe Preliminar. Revista Biomédica 9:224229, 1998.

4. Carmo M, TimenetzKy ST, Santos N, Gouvea V. Survey of rotavirus $G$ and $P$ types associated with human gastroenteritis in São Paulo, Brazil, from 1986 to 1992. Journal of Clinical Microbiology 32:2622-2624, 1994.

5. Cialet M, Estes MK, Conner ME. Comparative aminoacid sequence analysis of the outer capsid protein VP4 from four lapine rotavirus strain reveals identify with genotype $p$ human rotavirus. Archives of Virology 142:1059-1069, 1996.

6. Estes MK. Advances in molecular biology: impact on rotavirus vaccine development. Journal of Infectious Diseases 174:S37-46, 1996.

7. Fathy S, Kamal M, El-Maraghi S, Falthy A. Serotyping of group A rotavirus in Egyptian neonates and infants less than 1 year old with acute diarrhea. Journal of Clinical Microbiology 1996-2998, 1997.

8. Gonzáles-Losa MR, Puerto-Solís M, Polanco-Marín GG, Peniche-Rodriguez R, Puerto FI. Frecuencia de serotipos $\mathrm{G}$ de rotavirus aislados de niños com diarrea en mérida,
Yucatán, México. Revista de Investigaciones Clinicas 46:215-219, 1994.

9. Gouvea V, Ho MS, Galss R, Woods P, Forrester B, Robinson C, Ashley R, Ripenhoff-Talty M, Clark HF, Taniguchi K, Meddix E, McKellar B, Pickering L. Serotypes and electropherotypes of human rotavirus in USA: 19871989. Journal of Infection Diseases 162:362-367, 1990.

10. Gouvea V, Castro L, Timenetsky MC, Greenberg H, Santos N. Rotavirus serotype G5 associated with diarrhea in Brazilian children. Journal clinical Microbiology 32:1408-1409, 1994.

11. Holmes JL, Kirwood CD, Gerna G, Clemens JD, Rao MR, Naficy AB, Abu-Elayazeed R, Savarino SJ, Glass RI, Gentsch JR. Characterization of unusual G8 rotavirus strains isolated from Egyptian children. Archives of Virology 144:1381-1396, 1999.

12. Kikwood CD, Biashop RF, Coulson DS. Human rotavirus VP4 contains strain-specific and cross-reactive neutralizarion sites. Archives of Virology 141:587-560, 1996.

13. Kikwood CD, Gentsch JR, Hoshino Y, Clark HF, Glass IR. Genetic and antigenic characterization of serotype $\mathrm{P}(6) \mathrm{G} 9$ human rotavirus strain isolatted in the United States. Virology 256:45-53, 1999.

14. Maunula L, von Bonsdorff $\mathrm{CH}$. Short sequence define genetic lineages: phylogenetic analysis of group A rotavirus based on partial sequence of genoma segments 4 and 7. Journal of General Virology 79:321-332, 1998.

15. Offit PA. Immunologic determinants of protection against rotavirus diseases. EN: Rotaviruses. R:F. Raming. Spring Verlag, Alemanha, 1994.

16. Padilla L, Méndez E, Menchaca G, Contreras J, Romero P, Puerto F, Guiscafre H, Mota F, Herrera I, Cedillo R, Muñoz O, Calva J, Guerero ML, Coulson B, Greenberg HB, López S, Arias CF. Antigenic and genomic diversity 
of human rotavirus VP4 in two consecutive epidemic season in México. Journal of Clinical Microbiology 36:1688-1692, 1998.

17. Palombo FA, Clark R, Bishop RF. Characterization of "European-like" serotype G8 human rotavirus isolated in Australia. Journal Medical Virology 60:56-66, 2000.

18. Puerto FI, Polanco-Marín GG, Puerto-Solís MR, OrtegaAcosta G, Gongora-Biachi RA. Diarrea infantil aguda por rotavirus en una población pediátrica de Mérida, Yucatán. Boletín Médico del Hospital Infantil de México 46:171174, 1989.

19. Puerto MR. Monografia de rotavirus. Tesis de Especialidad. Yucatán. Universidad Autónoma de Yucatan, 1995.

20. Timenetsky MCST, Gouveo V, Santos N, Alge ME, Kisiellius JJ, Carmona RCC. Outbreak of severe gastroenteritis in adults and children associated with tyoe
G2 rotavirus. Journal Diarrhoeal Research 14:71-74, 1996.

21. Unicomb LE, Podder G, Gentsh JR, Woods PA, Hasan KZ, Faruque ASG, Albert MJ, Glass IR. Ecidence of highfrequency genomic reassortment of group $A$ rotavirus strains in Bangladesh: emergence of type G9 in 1995. Journal of Clinical Microbiology 37:1885-1891, 1999.

22. Ward RL. Mechanisms of protection against rotavirus in human and mice. Journal Infectious Diseases 174:551558, 1996.

23. Wu H, Taniguchi K, Urasawa T, Urasawa S. Serological and genomic characterization of human rotavirus detected in China. Journal Medical Virology 55:168-176, 1998.

24. Yasutaka $\mathrm{H}$, Kapikaian $\mathrm{A}$. Rotavirus vaccine development for the prevention of severe diarrhea in infants and young children. Trends in Microbiology 2:242-249, 1994. 